\title{
A Novel RFID Distance Bounding Protocol Based on Physically Unclonable Functions
}

\author{
Süleyman Kardaş ${ }^{1,2}$, Mehmet Sabir Kiraz ${ }^{1}$, \\ Muhammed Ali Bingöl ${ }^{1,3}$, and Hüseyin Demirci ${ }^{1}$ \\ 1 TUBITAK UEKAE, Gebze, Kocaeli, Turkey \\ 2 Sabanci University, Istanbul, TR-34956, Turkey \\ 3 Istanbul Technical University, \\ Institute of Science and Technology, Istanbul,Turkey
}

\begin{abstract}
Radio Frequency Identification (RFID) systems are vulnerable to relay attacks (i.e., mafia, terrorist and distance frauds) when they are used for authentication purposes. Distance bounding protocols are particularly designed as a countermeasure against these attacks. These protocols aim to ensure that the tags are in a distant area by measuring the round-trip delays during a rapid challenge-response exchange of short authenticated messages. Terrorist fraud is the most challenging attack to avoid, because a legitimate user (a tag owner) collaborates with an attacker to defeat the authentication system. Many RFID distance bounding protocols have been proposed recently, with encouraging results. However, none of them provides the ideal security against the terrorist fraud.

Motivated by this need, we first introduce a strong adversary model for Physically Unclonable Functions (PUFs) based authentication protocol in which the adversary has access to volatile memory of the tag. We show that the security of Sadeghi et al.'s PUF based authentication protocol is not secure in this model. We provide a new technique to improve the security of their protocol. Namely, in our scheme, even if an adversary has access to volatile memory she cannot obtain all long term keys to clone the tag. Next, we propose a novel RFID distance bounding protocol based on PUFs which satisfies the expected security requirements. Comparing to the previous protocols, the use of PUFs in our protocol enhances the system in terms of security, privacy and tag computational overhead. We also prove that our extended protocol with a final signature provides the ideal security against all those frauds, remarkably the terrorist fraud. Besides that, our protocols enjoy the attractive properties of PUFs, which provide the most cost efficient and reliable means to fingerprint chips based on their physical properties.
\end{abstract}

Keywords: RFID, Distance Bounding Protocol, PUF, Security, Terrorist fraud.

\section{Introduction}

Radio Frequency IDentification (RFID) is a technology that has been widely used in daily life, such as in access control, in electronic passports, public

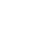


transportation, payment and ticketing. The reader communicates with the RFID tags using a wireless channel where the security and privacy requirements are satisfied via cryptographic building blocks (e.g, hash functions, symmetric encryptions and secure authentication protocols). However, such cryptographic mechanisms are not sufficient to enforce strong authentication in RFID systems. The seminal works of Desmedt et al. [8] and Beth et al. [3] on mafia and terrorist frauds demonstrated how an adversary can defeat such protocols by simply relaying the messages without dealing with cryptography. The chess grandmaster attack, which is introduced by Conway 7] in 1976, can be given as an illustration of the problem. In this problem, an unskilled player challenges two different chess grandmasters simultaneously. By only relaying the moves of the grandmasters the player finally either defeats one of the grandmasters or draws against both. Those kinds of attacks have been practically demonstrated in many different contexts and especially in RFID systems [13, 15 17, 22]. Nowadays, RFID and contactless smart card producers take relay attacks into account in the design of secure commercial products [26].

Mafia fraud is a kind of relay attack where an adversary is willing to be authenticated as if she is a legitimate prover. In order to perform this attack, the adversary relays the messages between a prover (e.g., a tag) and a verifier (e.g., a reader). Terrorist fraud is similar to mafia fraud except that the legitimate tag collaborates with an adversary to be able to authenticate her. However, the prover does not reveal his long-term private key to the adversary [6]. Finally, distance fraud is also similar to relay attacks where a fraudulent prover tries to persuade the verifier that she is within a certain authentication area whereas she is not.

In order to mitigate these frauds, two main countermeasures have been adopted. The first one is based on measuring the radio signal strength (RSS) so that the verifier can learn whether the prover is close to it. This method has a drawback that a capable adversary can regulate its signal strength to convince the verifier that it is close to the verifier [14]. The second one is measuring the round trip time of exchanged messages between the reader and the tag[8]. At Eurocrypt'93, Brands and Chaum [5] proposed the first distance-bounding protocol to prevent mafia fraud and distance fraud while leaving the terrorist fraud attack as an open issue. Then, several such protocols, which use the round trip time method, have been proposed to improve security levels against distance, mafia and terrorist attacks [2, 14, 20, 23, 25, 28, 30, 32, 33, 36]. However, one of the main obstacles of the existing distance bounding protocols is achieving the ideal security level (i.e., $(1 / 2)^{n}$ where $n$ is a security parameter) against terrorist fraud. Some attempts to thwart terrorist fraud [33] yield a more serious security problem namely, the key recovery attack. This attack occurs due to the misuse of long-term key in the protocol [20].

Our Contributions. In this paper, we first analyze the security of Sadeghi et al.'s PUF based RFID authentication protocol [29] by our stronger adversarial model in which an adversary has access to the volatile memory of the tag. We show that their protocol is not secure in this model and we propose a new 
technique to avoid this attack even if the adversary has the ability to access volatile memory.

Next, we apply this technique to propose a new PUF based RFID distance bounding protocol. To the best of our knowledge, this is the first paper that introduces a PUF based RFID distance bounding protocol. It is well-known that obtaining the long-term key of a tag is crucial in order to successfully perform the terrorist and the distance frauds. One of the main problems of existing distance bounding protocols is storing the long-term key into its memory which can be obtained by a fraudulent prover. Our protocol has the advantage that the longterm key will not be stored in the memory of the tag but will be reconstructed by using a PUF circuit.

Our first PUF based distance bounding protocol is based on the well-known Hancke and Kuhn's scheme [14] which is the starting point of this work. Although their original protocol is known to be simple and efficient, the adversary's probability of success is high (namely $(3 / 4)^{n}$ for both the distance and the mafia frauds, and 1 for the terrorist fraud). By the use of PUF, the adversarial capabilities of the terrorist fraud is reduced to that of the mafia fraud. In this way, we improve the security of Hancke-Kuhn's protocol against the terrorist fraud from 1 to $(3 / 4)^{n}$.

We also propose our second distance bounding protocol which is an extension of the first one involving a hash-based final signature. To the best of our knowledge, this is the first protocol that achieves the ideal security levels $(1 / 2)^{n}$ against all frauds.

Outline of the paper. The organization of the paper is as follows: In Section 2, we briefly describe some existing distance bounding protocols. In Section 3, we illustrate the notion of PUF functions and its characteristics. Section 4 describes the adversary capabilities for both PUFs and distance bounding protocols. In Section 5, we propose our first distance bounding protocol and analyze its security. In Section 6, we present our second protocol and analyze its security. Section 7 concludes the paper.

\section{Distance Bounding Protocols}

Distance bounding approach was a breakthrough to thwart relay attacks by measuring the round trip time of short authenticated messages. Brands and Chaum introduced the first distance bounding protocol [5]. This protocol aims to bring a solution to mafia and distance frauds. It consists of three phases, a slow phase, followed by a fast phase and a final signature phase. The first slow phase is used to exchange the committed random bits. The proximity verification is achieved by a bitwise challenge-response during the second phase (i.e., fast phase), namely after series of $n$ rounds where $n$ is a security parameter. For each round of the fast phase, the verifier measures the round-trip time in order to extract the propagation time. Finally, the prover sends a final signature to the verifier and opens the commitments to complete the protocol. The success 
probability of mafia and distance frauds for this protocol are $(1 / 2)^{n}$, but it is not secure against terrorist fraud.

Čapkun et al. modified the Brands and Chaum's protocol to achieve mutual authentication with distance-bounding [36]. However, their protocol is also vulnerable to terrorist fraud and is not resilient to bit errors during the rapid bit exchange.

Hancke and Kuhn proposed the first use of distance bounding protocol for RFID systems [14]. The major difference from Brands and Chaum's protocol is that it does not involve a final signature phase. This protocol involves a common secret symmetric-key $k$ between a prover and a verifier. This protocol can be briefly described as follows. The verifier first generates a nonce $N_{v}$ and sends it to the prover. Similarly, the prover also generates a nonce $N_{p}$ and sends it to the verifier. Two n-bit registers $R^{1}, R^{2}$ are computed such that $R^{1} \| R^{2}=$ $f\left(k, N_{v}, N_{p}\right)$ where $f$ is a public pseudorandom function. After that, $n$-round fast phase starts. For each $i$-th round, the verifier picks a random challenge-bit $c_{i}$ and sends it to the prover. The prover replies with a response-bit $r_{i}$ such that

$$
r_{i}=\left\{\begin{array}{ll}
R_{i}^{0} & \text { if } c_{i}=0 \\
R_{i}^{1} & \text { if } c_{i}=1
\end{array}\right\} .
$$

The success probabilities against the mafia fraud and distance fraud are both equal to $(3 / 4)^{n}[14,19]$.

Distance bounding protocols are classified into two classes depending on whether a final signature is involved (e.g., [2, 19, 20, 23, 25, 32, 33]). These papers mostly focused on improving the security against mafia and distance frauds, and in fact some of them achieved the ideal security level $(1 / 2)^{n}$ against only both frauds. Furthermore, some others achieve $(3 / 4)^{n}$ as the best security level for terrorist fraud. Unfortunately, none of these protocols achieve the ideal security against terrorist fraud.

Avoine et al. [1] introduced a unified framework for improving the analysis and the design of distance bounding protocols. The black-box and the whitebox security models are introduced in the distance bounding domain, and the relation between the frauds are described with respect to these models. In the white-box model, the prover can provide more information to the adversary since the can access the internal key. We note that all the protocols in the literature are analyzed in the white-box model and therefore, the security level is worse than that can be achievable in the black-box model.

In the next section, PUFs will be described which will be later used in our protocols. We later show that the use of PUFs eliminates our protocols to be analyzed in the white-box model.

\section{Physically Unclonable Functions (PUFs)}

Physically Unclonable Functions (PUFs) were invented by Naccache and Fremanteau in 1992 [24]. A PUF is defined as an unclonable function that maps 
challenges to responses. The response $r$ is calculated as a result of physical properties such as delays of gates and wires in a circuit, variations in the temperature and supply voltage. The unclonability of the function is guaranteed as a result of these physical processes. An ordinary PUF circuit may produce slightly different outputs to the same inputs. Using mechanisms like Fuzzy Extractors [10, 37], one can guarantee that PUF circuit produces the same response to the same challenge. For further information about other types of PUFs we refer to [31].

Since PUFs behave as a random function, it is hard to predict the inputs as given the outputs. Therefore, they can also be considered as one-way functions. In addition, a PUF circuit can easily be implemented into a small area with less than 1000 gates [34]. Besides that, their intrinsic structure yields resistance against tampering. When the adversary tries to evaluate a PUF or an IC, for instance, by using the probes to measure the wire delays, the characteristics of that particular PUF will be changed. Thus, this physical attack will not give any advantage to the adversary 21]. These features make PUF an attractive tool for authentication mechanisms in RFID systems.

In [27] the use of PUFs in RFID systems is proposed. The idea is to use a set of predetermined set of challenge-response pairs with the help of a database. The readers use the database to identify the tag. In this protocol, the possible challenge-response pairs are restricted with the database. A challenge also cannot be used for the second time, since it results to replay attacks. The proposed scheme has been implemented and analyzed in [9].

In [34], PUF is used as a secure key derivation mechanism. Instead of putting the key in memory, it is derived from the circuit each time whenever it is required. This property of PUFs mitigates the hardware-based cloning attacks. A practical illustration is RFID tags, which can easily be cloned. When equipped with a PUF, creating a clone in a reasonable time is impractical. Furthermore, the concept of SRAM-PUFs is proposed in [18]. They propose that SRAM memory cells can be used as a PUF mechanism which are readily available in the existing RFID chips.

A simple privacy preserving identification system is proposed in [4]. This protocol uses a PUF $P$ for frequently updating the identity of tags where the reader stores the vector $\left(I D, P(I D), P^{2}(I D), \ldots, P^{k}(I D)\right)$. To authenticate to a reader, a tag first sends its current $I D$ and updates it using the PUF $P(I D)$. The reader searches the current identifier of the tag from the database. If the reader finds a tuple, it authenticates the tag and removes all the elements which have been used before in the authentication mechanism. Note that this protocol suffers from the Denial of Service Attacks since the tag must be re-initialized after at most $k$ sessions.

Sadeghi et al. 29] suggested to deploy PUF (in a similar way as described by Tuyls and Batina in [34]) in order to develop a privacy-preserving tag authentication protocol for RFID systems. This protocol provides destructive privacy in the Vaudenay's formal framework [35]. 
In this paper, we will focus on an ideal PUF $P$ such that $P:\{0,1\}^{\ell} \rightarrow\{0,1\}^{m}$ where the challenge $c_{i}$ is mapped to the response $r_{i} . P$ is said to be an ideal PUF if the following properties are satisfied.

1. If $c_{i}=c_{j}$, then we have $r_{i}=r_{j}$ for a PUF on a particular device. Presenting the same challenge to the PUF on a different device will produce a different response.

2. The mapping between $c_{i}$ and $r_{i}$ is unpredictable and random. For instance, if $r_{i}$ and $r_{j}$ differ in only a single bit, knowledge of $c_{i}$ does not reveal usable information to predict $c_{j}$.

3. Any attempt to physically tamper with the device implementing $P$ causes to change its physical characteristics. Namely, $P$ is then destructed and can no longer be evaluated correctly.

We note that the third property of the idealized PUF can be achieved by integrating PUF circuit with the chip on the tag. To do so, Tuyls et al. in [34] propose Integrated PUFs (I-PUFs). For further information we recommend reading [29, 34]. In this work, we use the ideal PUF for distance bounding protocols and show how the security is enhanced to ideal levels.

\section{Adversary Capabilities}

In this section, we first present a stronger adversarial model for analysis of PUF based RFID authentication protocols which considers the accessibility to the internal state of tags. We next discuss the notion of white and black box models for distance bounding protocols. We aim to unify and express the adversarial capabilities of PUFs and distance bounding protocols.

\subsection{Adversary Capabilities for PUFs}

In a PUF based authentication protocol, the shared secrets are stored in its physical characteristics instead of storing them in a non-volatile memory. These keys are reconstructed whenever needed during the execution of the protocol. As soon as the keys are reconstructed, they are stored in a volatile memory of the RFID chip. In some previous articles (e.g., 29, 34]), it is assumed that the communication between a PUF circuit and a chip is not tractable by any side-channel attack.

Unlike the previous works, in this paper, we propose a more stronger adversary model where an attacker has the ability to compromise the tag and reaches the state in the volatile memory. Since the structure of the PUF circuit has been destroyed, the attacker is no longer able to re-evaluate the PUF again. Thus, a malicious tag owner can perform only one side-channel attack on the tag and access the volatile memory only once. For instance, Halderman et al. recently demonstrated a side-channel attack for DRAM, called cold boot attack [12]. In this attack, they first powered off the system and later showed how to extend the main memory persistence by 'freezing' the DRAM chips in order to maintain the 
memory cell state. In this way, an adversary will be able to retrieve any password or cryptographic key that was not disappeared before the system is switched off.

The protocol of Sadeghi et al. 29] is facing a similar attack described above. Their protocol is briefly described as follows (Figure 1). Let $l \in \mathbb{N}$ be a security parameter, and $\mathrm{F}:\{0,1\}^{k} \times\{0,1\}^{2 \alpha} \rightarrow\{0,1\}^{\beta}$ be a public pseudorandom (PRF) function. Each tag $\mathcal{T}$ is equipped with a PUF function $\mathrm{P}:\{0,1\}^{\gamma} \rightarrow\{0,1\}^{k}$ and is initialized with a random state $S_{1} \in_{R}\{0,1\}^{\gamma}$. The credential of each tag (ID, $K)$, where $K \leftarrow P(S)$ and is stored in the database DB of the reader. The reader $\mathcal{R}$ first picks a random nonce $a$ to the tag $\mathcal{T}_{I D}$. Then, $\mathcal{T}_{I D}$ picks a random nonce $b$ and evaluates the PUF function $K=P(S)$. $\mathcal{T}_{I D}$ computes $c=F_{K}(a, b)$ and sends the message $c$ along with the random nonce $b$ and immediately erases $K, a, b$ and $c$ from its volatile memory. Upon receiving of $b$ and $c, \mathcal{R}$ evaluates $c^{\prime}=F_{K}(a, b)$ for each tuple $(I D, K)$ in DB until there is a match. If a matching $(I D, K)$ is found, then it accepts $\mathcal{T}_{I D}$ and returns $I D$; otherwise, it rejects by sending $\perp$ back.

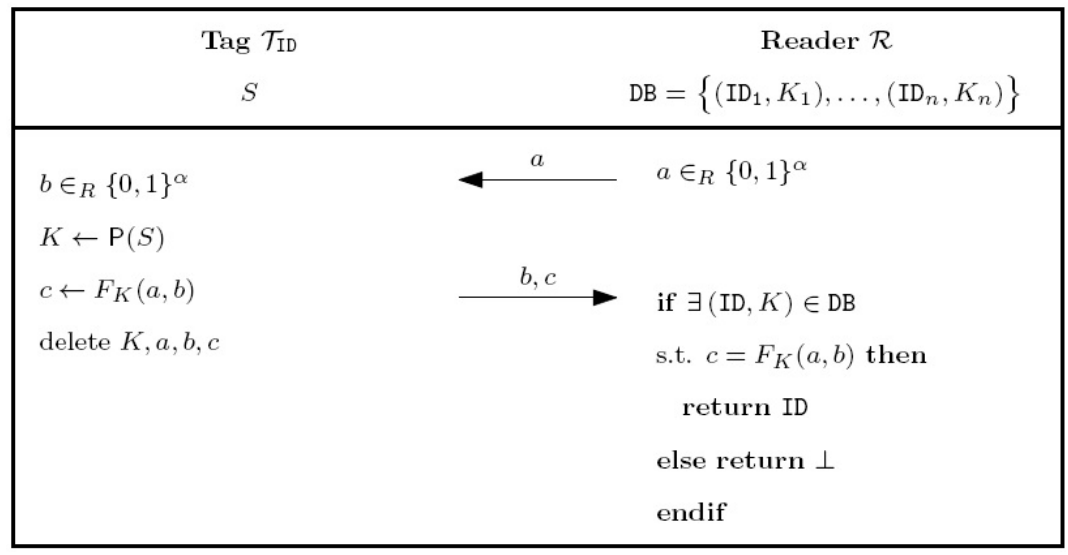

Fig. 1. Sadeghi et al.'s authentication protocol 29]

The authors claim that their protocol achieves destructive-privacy under the assumption that $K$ is inaccessible. However, we show that their protocol suffers from the same above-mentioned cold-boot attack. Assume that an adversary sends a random nonce $a$ to the $\operatorname{tag} \mathcal{T}_{I D}$. $\mathcal{T}_{I D}$ then generates another random nonce $b$ and reconstructs a secret $K$ by evaluating the PUF with input $S$. The secret $K$ is stored in the volatile memory during the computation of $c=F_{K}(a, b)$. The adversary compromises $\mathcal{T}_{I D}$ while $c=F_{K}(a, b)$ is computed and can capture the secret $K$. Hence, the tag can be successfully cloned although the structure of the PUF circuit has been destroyed.

In order to thwart this attack, instead of using only one key we propose to use two different keys $K, L$ which are consecutively generated as outputs of the PUF function. Note that $K$ and $L$ never appear in the volatile memory at the 
same time. First, $K$ is used as an input of one-way PRF function, and then completely deleted from the memory. Next, in a similar way, $L$ is generated and used in the PRF function. Hence, whenever an adversary applies the abovementioned attack he will be able to obtain only one of the keys, and hence will not have sufficient information to defeat the privacy. Also, since the PUF circuit has been destroyed he will not be able to perform the same attack again. Thus, applying our technique avoids the tag cloning.

\subsection{Adversary Capabilities for Distance Bounding Protocols}

In the analysis of our protocols, Dolev-Yao adversary model are considered [11]. In this model, the adversary can perform polynomial number of computations and cannot obtain the secret keys from the honest parties. This assumption is then relaxed with the terrorist and distance frauds, where the prover has access to the keys [1]. However, he disagrees to share these keys with any third party. The adversary may use one of the three strategies to query a prover such as pre-ask strategy, post-ask strategy and early-reply strategy. The detailed explanations of these strategies are addressed in [1].

As in the conventional distance bounding protocols, we also assume that the verifier is an honest party where it faithfully follows the protocol specifications without cheating. Mafia fraud is a kind of man-in-the-middle attack where an adversary defeats both honest parties i.e., verifier and prover. Unlike mafia fraud, in distance and terrorist frauds, the prover himself is dishonest. The previous distance bounding protocols consider that the prover has a full control on the execution of the algorithm in the device. As it is discussed in Section 3 . PUFs can be used to provide resistance against side-channel attacks. Therefore, an adversary can be limited to the execution of the algorithm inside the device. In order to analyze distance bounding protocols, the generic capabilities of the adversary are addressed in [1]. The capabilities are categorized in two models, white-box model and black-box model. The following definitions of these two models are excerpted from [1].

Definition 1. (Black-box model) In this model, the prover cannot observe or tamper with the execution of the algorithm.

Definition 2. (White-box model) In this model, the prover has full access to the implementation of the algorithm and has a complete control over the execution environment.

Regarding to the white-box and the black-box models Figure 2 presents the relation between the distance, mafia and terrorist frauds. An arrow from $X$ to $Y$ means that, for any fraud in $X$ that succeeds with probability $p_{X}$, then there exists an attack in $Y$ that succeeds with probability $p_{Y}$ such that $p_{Y} \geq p_{X}$. Two side arrow means that the success probabilities of two corresponding frauds are equal [1].

It is interesting to note that in the black-box model, the success probabilities of the mafia and the terrorist frauds are equal (Figure 2). 


\section{Black-box model White-box model}

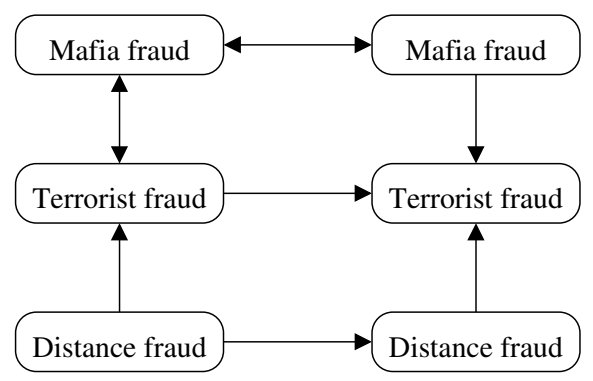

Fig. 2. Relations between the frauds in the white-box and the black-box models [1]

\section{Our First Protocol}

We now propose the first PUF based distance bounding protocol which is efficient for implementation in low cost devices. In the next section, we extend this protocol by adding a final signature to enhance the security against terrorist fraud. The former achieves the security level of $(3 / 4)^{n}$ against mafia, terrorist and distance frauds, where $n$ is the number challenge/response bits during the fast phase. We show in the next section that the latter achieves the ideal security level against all the frauds (i.e., $\left.(1 / 2)^{n}\right)$.

\subsection{Protocol Descriptions}

Our first distance bounding protocol is based on Hancke and Kuhn's scheme [14], which is the starting point of this work. Although their protocol is simple and efficient the adversary's probability of success is high. The steps of our protocol are summarized below and depicted in Figure 3.

Initialization. Let $P_{i}:\{0,1\}^{k} \rightarrow\{0,1\}^{\ell}$ be a (unique) ideal PUF of the $i$ th legitimate prover $\mathcal{P}_{i}$. The credentials database DB of the verifier $\mathcal{V}$ stores a tuple $\left(K_{i}, L_{i}\right)$ where $K_{i}=P_{i}\left(G_{i}^{1}\right)$ and $L_{i}=P_{i}\left(G_{i}^{2}\right)$ for random states $G_{i}^{1}, G_{i}^{2} \in R$ $\{0,1\}^{k}$. Let also $F:\{0,1\}^{\ell} \times\{0,1\}^{2 \ell} \rightarrow\{0,1\}^{2 \ell}$ be a one-way pseudorandom function. We denote $n$ as the main security parameter of the fast phase where $3 n=2 \ell .|S|$ denotes the bit-length of a bit-string $S$.

Our protocol consists of two phases: a slow phase and a fast phase.

\section{Slow phase:}

- First of all, $\mathcal{V}$ generates a random nonce $r_{V}$ and sends it to $\mathcal{P}_{i}$.

- Upon receiving $r_{V}, \mathcal{P}_{i}$ generates a random nonce $r_{P}$ and reconstructs $K_{i}=$ $P_{i}\left(G_{i}^{1}\right) . \mathcal{P}_{i}$ computes $T=F_{K_{i}}\left(r_{P}, r_{V}\right)$, then immediately deletes $K_{i}$ from the memory. After that, $\mathcal{P}_{i}$ reconstructs the secret key $L_{i}=P_{i}\left(G_{i}^{2}\right)$ and computes the message $F_{L_{i}}(T)$. Similarly, $\mathcal{P}_{i}$ immediately deletes $L_{i}$ from 
the memory. The value $F_{L_{i}}(T)$ is divided into three registers $v_{1}, v_{2}$ and $v_{3}$ where $\left|v_{1}\right|=\left|v_{2}\right|=\left|v_{3}\right|=n$. Finally, $\mathcal{P}_{i}$ sends $r_{T}$ and $v_{1}$ to $\mathcal{V}$.

- Upon receiving $r_{T}$ and $v_{1}$, for each tuple $\left(K_{i}, L_{i}\right)$ in DB $\mathcal{V}$ searches $v_{1}^{\prime}, v_{2}^{\prime}, v_{3}^{\prime}=$ $F_{L}\left(F_{K}\left(r_{P}, r_{V}\right)\right)$ such that $v_{1}^{\prime}=v_{1}$. If not found, $\mathcal{V}$ aborts the protocol.

\section{Fast phase:}

- The fast phase consists of $n$ bitwise challenge-response exchange. For each round $j \in\{1, \ldots, n\}, \mathcal{V}$ picks a random challenge bit $c_{j}$ and sends it to $\mathcal{P}_{i}$.

$-\mathcal{P}_{i}$ immediately responds $r_{j}=v_{2}^{j}$ if $c_{j}=0$, otherwise $r_{j}=v_{3}^{j}$.

Verification. Whenever the fast phase is finished $\mathcal{V}$ verifies that the responses from $\mathcal{P}_{i}$ are correct and checks whether $\Delta t_{j} \leq \triangle t_{\max } \forall j=1, \ldots, n$ where $\triangle t_{\max }$ is a timing bound.

\subsection{Security Analysis}

Mafia, terrorist, and distance frauds are the three main security concerns when considering distance bounding protocols. The following Theorem 1 indicates that no adversary (e.g., a malicious tag owner) can access to both secrets $K_{i}$ and $L_{i}$. Thus, the use of PUF in the protocol makes the RFID tags as tamper proof against any malicious adversary.

Theorem 1. Let $K_{i}, L_{i}$ be secrets of a tag $\mathcal{T}_{i}$ for some $i$ in the above-mentioned protocol (see Figure 3). Assume that there is an adversary $\mathcal{A}$ with a full sidechannel capability on the tag $\mathcal{T}_{i}$. If $P_{i}$ is an ideal PUF, then $\mathcal{A}$ can only access either the secret $K_{i}$ or the secret $L_{i}$, but not both in the same tag $\mathcal{T}_{i}$.

Proof. (sketch) The pre-keys $G_{i}^{1}$ and $G_{i}^{2}$ are feeded into the $P_{i}$ function to generate the real keys $K_{i}$ and $L_{i}$. The real keys only appear during the execution of the protocol. Note that $K_{i}$ and $L_{i}$ never appear in the memory of $\mathcal{T}_{i}$ at the same time because $K_{i}$ is first used as an input of a one-way PRF function, and then completely deleted from the memory. Next, in a similar way, $L_{i}$ is generated and used in the PRF function. Whenever $\mathcal{A}$ applies a side channel attack to $\mathcal{T}_{i}$, the physical characteristics of the PUF $P_{i}$ will be broken and will no longer be evaluated correctly. If $\mathcal{A}$ applies side-channel attack to extract $K_{i}$ then the structure of $P_{i}$ will be destroyed and $L_{i}$ cannot be generated. Similarly, if $\mathcal{A}$ applies side-channel attack to extract $L_{i}$ she cannot obtain $K_{i}$ since it is already deleted. Therefore, $\mathcal{A}$ can access either $K_{i}$ or $L_{i}$ but not both. Hence, $\mathcal{A}$ will not be able to get the complete key of $\mathcal{T}_{i}$.

Theorem 1 indicates that a malicious prover cannot obtain the secret keys, and thus cannot evaluate the registers $v_{1}, v_{2}, v_{3}$. Unlike existing distance bounding protocols, it is not possible to apply the white-box analysis to our protocol. Therefore, we analyze our protocol according to the black-box model. In the black-box model, note that it is already proven that the capability of terrorist 


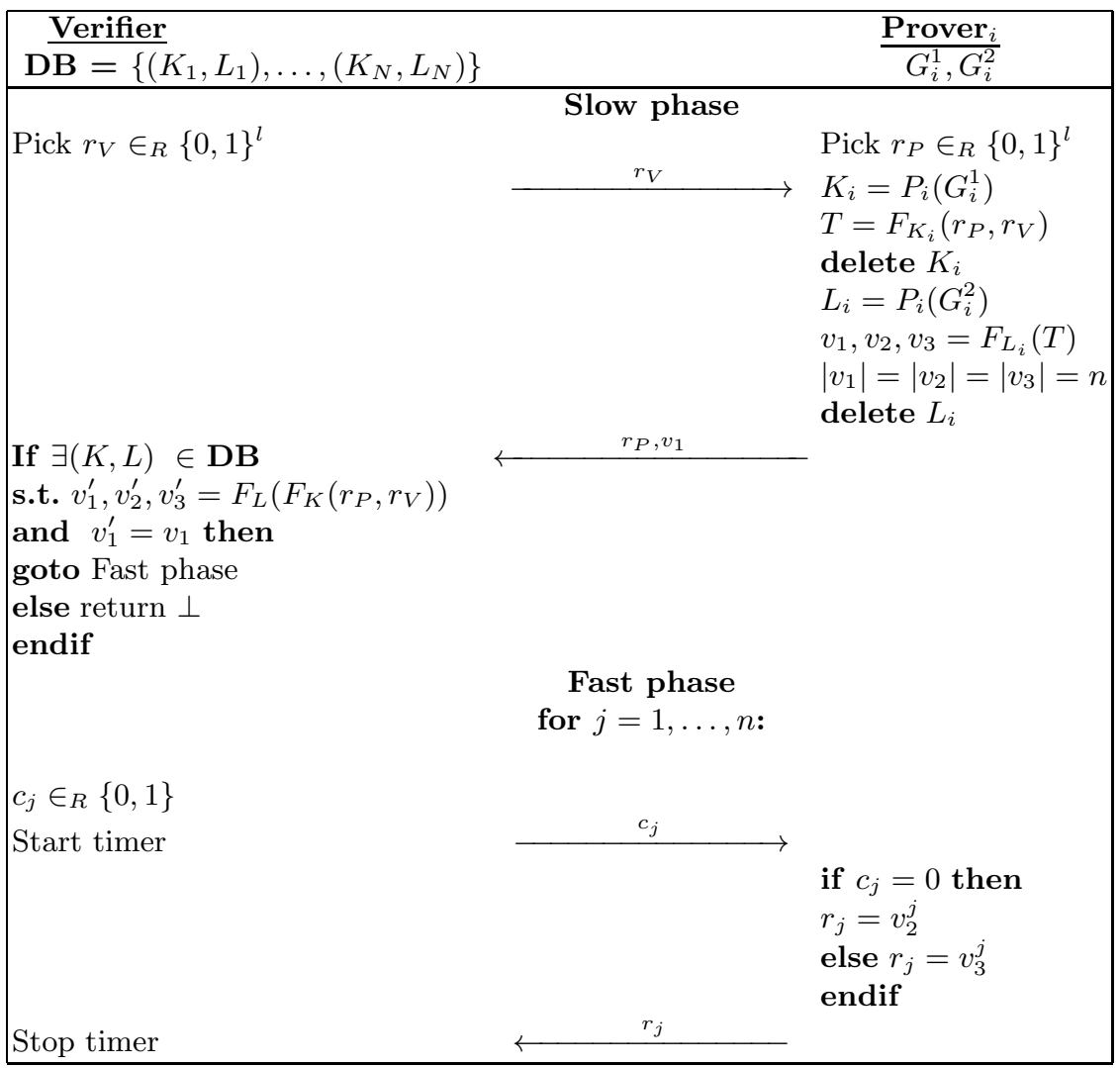

Fig. 3. Our first PUF based distance bounding protocol without a final signature

fraud is equivalent to the mafia fraud [1] (see also Figure 2). Hence, we combine the security analysis of both mafia and terrorist frauds.

Note that a malicious prover can access the registers $v_{1}, v_{2}, v_{3}$ by applying side-channel attack only once. Furthermore, he can complete only the current session successfully because of the destruction of PUF. However, since the registers $v_{1}, v_{2}, v_{3}$ are randomized this does not give any future advantage to the adversary.

For a distance bounding protocol, an adversary is able to use three different strategies to conduct her attack such that early-reply, pre-ask, and post-ask [1]. We denote by $\mathcal{A}$ a malicious adversary. Let also denote by $M F, T F$ and $D F$ the mafia fraud, the terrorist fraud and the distance fraud, respectively. Let $F$ be a fraud and $S$ be the strategy used by the adversary $\mathcal{A}$. Let $\operatorname{Pr}_{F \mid S}$ be the success probability in the black-box model of the fraud $\mathrm{F}(M F / D F / T F)$ using the strategy $\mathrm{S}$ (early/pre/post). Note that the strategies can also be combined and this is denoted by an \&. Next, we describe the success probability of each fraud as follows. 
Mafia and terrorist fraud analysis. The adversary uses pre-ask or post-ask strategies in order to achieve mafia or terrorist fraud.

Pre-ask strategy [1]. In this strategy, $\mathcal{A}$ first relays the slow phase between $\mathcal{V}$ and $\mathcal{P}$. Then $\mathcal{A}$ executes the fast phase with $\mathcal{P}$. $\mathcal{A}$ sends predicted challenges $c_{j}^{\prime}$ to $\mathcal{P}$ and get the responses $r_{j}^{\prime}$ corresponding to her challenges. With this a strategy, $\mathcal{A}$ obtains only one of the register. Afterward, $\mathcal{A}$ executes the fast phase with $\mathcal{V}$ and receives the challenges $c_{j}$ s. There are two equal likely cases, (i) if $c_{j}=c_{j}^{\prime}$ $\mathcal{A}$ sends the correct response with probability of 1 ; otherwise, (ii) $\mathcal{A}$ guess the response with probability of $1 / 2$. Hence, the success probability of mafia fraud and terrorist fraud for $n$-round fast phase is computed as follows.

$$
\operatorname{Pr}_{M F \mid p r e}=P r_{T F \mid p r e}=\left(\frac{1}{2} \cdot 1+\frac{1}{2} \cdot \frac{1}{2}\right)^{n}=\left(\frac{3}{4}\right)^{n} .
$$

Post-ask strategy [1]. In pre-ask strategy, $\mathcal{A}$ first relays the slow phase, then executes the fast phase with $\mathcal{V}$. The probability of sending a correct response for a challenge is $1 / 2$. Then, $\mathcal{A}$ queries $\mathcal{P}$ with the correct challenges received during the fast phase to check whether she is succeed on cheating. The success probability of mafia fraud for this strategy is:

$$
\operatorname{Pr}_{M F \mid p o s t}=P r_{T F \mid p o s t}=\left(\frac{1}{2}\right)^{n} .
$$

To maximize the success probability the attacker chooses the best strategy. Hence, the success probability of both mafia and terrorist frauds are $(3 / 4)^{n}$.

Distance fraud analysis. In distance fraud, the tag owner herself is fraudulent who tries to cheat on her proximity from $\mathcal{V}$. It is important to highlight that unlike the existing protocols, the tag owner cannot control the internal executions of the tag in our protocol. The fraudulent prover can query its tag to get the responses. In distance fraud, since the prover is outside of the legal authentication region she should send the responses earlier in order to pass the proximity check (i.e., round trip time measurement). This is called early-reply strategy [1]. To ease our analysis, we denote the fraudulent tag owner by $\mathcal{A}$, and the tag by $\mathcal{T}$.

Pre-ask combined with early-reply strategy. In this strategy, $\mathcal{A}$ first relays the slow phase between $\mathcal{V}$ and $\mathcal{T}$, then executes the fast phase with $\mathcal{T}$. $\mathcal{A}$ can only obtain $n$-bit responses corresponding to her predicted challenges. Since $\mathcal{A}$ is not inside the neighborhood of $\mathcal{V}$, she sends her responses in advance. Two cases occurs for each round of the fast phase. (i) $\mathcal{A}$ predicts $\mathcal{V}$ 's challenge correctly, then she sends a correct corresponding response in advance. (ii) $\mathcal{A}$ cannot not predict $\mathcal{V}$ 's challenge correctly, but she sends a correct answer with probability of $1 / 2$. Thus, the distance fraud success probability is:

$$
\operatorname{Pr}_{D F \mid \text { pre\&early }}=\left(\frac{1}{2} \cdot 1+\frac{1}{2} \cdot \frac{1}{2}\right)^{n}=\left(\frac{3}{4}\right)^{n} .
$$


Post-ask combined with early-reply strategy. Similar to the mafia fraud analysis, it is clear that using the post-ask strategy is equivalent to randomly guessing the responses,

$$
\operatorname{Pr}_{D F \mid \text { post\&early }}=\left(\frac{1}{2}\right)^{n} \text {. }
$$

The distance fraud attacker chooses the strategy with the maximum success probability. Consequently, the success probability of distance fraud is $(3 / 4)^{n}$.

\section{Our Extended Protocol}

We are now ready to propose our extended protocol which is resistant to all the frauds.

\subsection{Protocol Descriptions}

In what follows, we present our second protocol which is an extension of the first one by adding a final signature. This protocol consists of three phases. The first two phases are exactly the same with the previous protocol. In the third phase, the prover computes the following final signature

$$
f_{\text {sign }}=h\left(c_{1}, \ldots, c_{n}, T, L_{i}\right) .
$$

where $h$ denotes a collusion resistant and one-way hash function. To evaluate $f_{\text {sign }}$, first prover regenerate $L_{i}$ once more and delete it from the memory as soon as $f_{\text {sign }}$ is computed. The prover sends $f_{\text {sign }}$ to the verifier, then the verifier checks the correctness of this message.

\subsection{Security Analysis}

In the first protocol, mafia and distance frauds can successfully pass the fast phase with probability of $(3 / 4)^{n}$ by predicting the challenges. However, this attack does not work in the extended protocol because the challenges received by the tag are digested in $f_{\text {sign }}$. In order to pass the authentication, the adversary must send a valid final signature to the verifier. Similar to the first protocol, there are two strategies for both mafia and terrorist frauds:

(i) In the pre-ask strategy, the adversary first executes the fast phase with the prover by sending $c_{1}^{\prime}, \ldots, c_{n}^{\prime}$ challenges, then prover replies with the corresponding responses $r_{1}^{\prime}, \ldots, r_{n}^{\prime}$. In the final phase, the adversary gets $f_{\text {sign }}^{\prime}=$ $h\left(c_{1}^{\prime}, \ldots, c_{n}^{\prime}, T, L_{i}\right)$. The final signature is valid if and only if all the challenges $c_{1}, \ldots, c_{n}$ sent by the verifier are equal to the ones predicted by the adversary. Thus, it is clear that the probability of $f_{\text {sign }}=f_{\text {sign }}^{\prime}$ is equal to $(1 / 2)^{n}$.

(ii) In the post-ask strategy, the adversary first plays with the verifier and guesses all the responses during the fast phase. If she passes the fast phase then it is easy to get the valid final signature from the prover by forwarding the 
challenges of the verifier. However, the probability of guessing all the correct responses during the fast phase is equal to $(1 / 2)^{n}$. Thus,

$$
\operatorname{Pr}_{M F}=\operatorname{Pr}_{T F}=\left(\frac{1}{2}\right)^{n} \text {. }
$$

Similarly, the security of the extended protocol for distance fraud is also bounded by $(1 / 2)^{n}$ due to the same reasons described-above. Namely, in order to receive a valid final signature from the tag the fraudulent prover should have queried the tag with all correct challenges in advance. Hence, the use of final signature enhances the security level of our extended protocol against the distance fraud to the ideal level $(1 / 2)^{n}$.

\section{Conclusion}

Relay attacks are indeed practical threats for RFID systems since using only cryptographic primitives it is not easy to thwart mafia, distance and terrorist frauds. Distance bounding protocols are used to mitigate these threats. However, the existing distance bounding protocols cannot achieve ideal security level against all frauds.

In this paper, we present the first PUF based distance bounding authentication protocol. Note that the protocols based on PUFs are known to be powerful since attacks can be easily prevented and the use of expensive cryptographic primitives can be minimized. In our protocol, we use the idea of key storage mechanism based on PUFs for public-key cryptosystems presented by Tuyls and Batina [34] (which is also later used for symmetric key storage by Sadeghi et al. 29] ). We modified their protocol in such a way that all the keys are not constructed at the same time. This enables us to achieve a stronger assumption and there is no way to extract the whole secret key from the tag. We show that our first protocol achieves the security level of $(3 / 4)^{n}$ against mafia, terrorist and distance frauds. We also extend our protocol by adding a final signature to enhance the security levels. Namely, we achieve the security level $(1 / 2)^{n}$ against for all mafia, terrorist and distance frauds. To the best our knowledge, this is the first paper that achieves the ideal security level $(1 / 2)^{n}$ against all frauds.

An interesting further question is whether it is possible to find an efficient protocol without a final signature having the ideal security level against all frauds.

\section{References}

1. Avoine, G., Bingöl, M.A., Kardaş, S., Lauradoux, C., Martin, B.: A Framework for Analyzing RFID Distance Bounding Protocols. Journal of Computer Security - Special Issue on RFID System Security 19(2), 289-317 (2011)

2. Avoine, G., Floerkemeier, C., Martin, B.: RFID Distance Bounding Multistate Enhancement. In: Roy, B., Sendrier, N. (eds.) INDOCRYPT 2009. LNCS, vol. 5922, pp. 290-307. Springer, Heidelberg (2009) 
3. Beth, T., Desmedt, Y.: Identification Tokens - or: Solving the Chess Grandmaster Problem. In: Menezes, A., Vanstone, S.A. (eds.) CRYPTO 1990. LNCS, vol. 537, pp. 169-177. Springer, Heidelberg (1991)

4. Bolotnyy, L., Robins, G.: Physically unclonable function-based security and privacy in rfid systems. In: Proceedings of the Fifth IEEE International Conference on Pervasive Computing and Communications, pp. 211-220. IEEE Computer Society, Washington, DC, USA (2007)

5. Brands, S., Chaum, D.: Distance Bounding Protocols. In: Helleseth, T. (ed.) EUROCRYPT 1993. LNCS, vol. 765, pp. 344-359. Springer, Heidelberg (1994)

6. Bussard, L., Bagga, W.: Distance-bounding proof of knowledge to avoid real-time attacks. In: Ryoichi, S., Sihan, Q., Eiji, O. (eds.) Security and Privacy in the Age of Ubiquitous Computing, Chiba, Japan. IFIP International Federation for Information Processing, vol. 181, pp. 223-238. Springer, Heidelberg (2005)

7. Conway, J.H.: On Numbers and Games. London Mathematical Society Monographs, vol. 6. Academic Press, London (1976)

8. Desmedt, Y., Goutier, C., Bengio, S.: Special Uses and Abuses of the Fiat Shamir Passport Protocol. In: Pomerance, C. (ed.) CRYPTO 1987. LNCS, vol. 293, pp. 21-39. Springer, Heidelberg (1988)

9. Devadas, S., Suh, E., Paral, S., Sowell, R., Ziola, T., Khandelwal, V.: Design and Implementation of PUF-Based "Unclonable" RFID ICs for Anti-Counterfeiting and Security Applications. In: 2008 IEEE International Conference on RFID, pp. 58-64 (2008)

10. Dodis, Y., Ostrovsky, R., Reyzin, L., Smith, A.: Fuzzy extractors: How to generate strong keys from biometrics and other noisy data. SIAM J. Comput. 38(1), 97-139 (2008)

11. Dolev, D., Yao, A.C.-C.: On the security of public key protocols. IEEE Transactions on Information Theory 29(2), 198-207 (1983)

12. Halderman, J.A., Schoen, S.D., Heninger, N., Clarkson, W., Paul, W., Calandrino, J.A., Feldman, A.J., Appelbaum, J., Felten, E.W.: Lest we remember: cold-boot attacks on encryption keys. Commun. ACM 52, 91-98 (2009)

13. Hancke, G.: A Practical Relay Attack on ISO 14443 Proximity Cards (February 2005) (manuscript)

14. Hancke, G., Kuhn, M.: An RFID Distance Bounding Protocol. In: Conference on Security and Privacy for Emerging Areas in Communication Networks - SecureComm 2005, Athens, Greece. IEEE (2005)

15. Hancke, G.P., Mayes, K., Markantonakis, K.: Confidence in smart token proximity: Relay attacks revisited. Computers \& Security 28(7), 615-627 (2009)

16. Hancke, G.P.: Practical Attacks on Proximity Identification Systems (Short Paper). In: IEEE Symposium on Security and Privacy - S\&P 2006, Oakland, California, USA. IEEE, IEEE Computer Society (2006)

17. Hlaváč, M., Rosa, T.: A Note on the Relay Attacks on e-Passports: The Case of Czech e-Passports. In: Cryptology ePrint Archive, Report 2007/244 (2007)

18. Holcomb, D.E., Burleson, W.P., Fu, K.: Initial SRAM state as a fingerprint and source of true random numbers for RFID tags. In: Proceedings of the Conference on RFID Security (2007)

19. Kara, O., Kardaş, S., Bingöl, M.A., Avoine, G.: Optimal Security Limits of RFID Distance Bounding Protocols. In: Ors Yalcin, S.B. (ed.) RFIDSec 2010. LNCS, vol. 6370, pp. 220-238. Springer, Heidelberg (2010)

20. Kim, C.H., Avoine, G., Koeune, F., Standaert, F.-X., Pereira, O.: The Swiss-Knife RFID Distance Bounding Protocol. In: Lee, P.J., Cheon, J.H. (eds.) ICISC 2008. LNCS, vol. 5461, pp. 98-115. Springer, Heidelberg (2009) 
21. Kulseng, L.: Lightweight mutual authentication, ownership transfer, and secure search protocols for rfid systems. Master's thesis, Electrical \& Computer Engineering Department, Iowa State University (2009)

22. Markantonakis, K., Tunstall, M., Hancke, G., Askoxylakis, I., Mayes, K.: Attacking smart card systems: Theory and practice. Information Security Technical Report 14(2), 46-56 (2009); Smart Card Applications and Security

23. Munilla, J., Peinado, A.: Distance bounding protocols for RFID enhanced by using void-challenges and analysis in noisy channels. Wireless Communications and Mobile Computing 8(9), 1227-1232 (2008)

24. Naccache, D., Fremanteau, P.: Unforgeable identification device, identification device reader and method of identification. Patent-EP0583709 (1994)

25. Nikov, V., Vauclair, M.: Yet Another Secure Distance-Bounding Protocol. In: Cryptology ePrint Archive, Report 2008/319 (2008)

26. NXP. NXP mifare plus - benchmark security for mainstream applications (2009), http://mifare.net/downloads/NXP_Mifare_Plus_leaflet.pdf

27. Ranasinghe, D.C., Engels, D.W., Cole, P.H.: Security and Privacy: Modest Proposals for Low-Cost RFID Systems. In: Proc. Auto-ID Labs Research Workshop Systems (2004)

28. Reid, J., Gonzaez Neito, J., Tang, T., Senadji, B.: Detecting relay attacks with timing based protocols. In: Bao, F., Miller, S. (eds.) Proceedings of the 2nd ACM Symposium on Information, Computer and Communications Security - ASIACCS 2007, Singapore, Republic of Singapore, pp. 204-213. ACM (March 2007)

29. Sadeghi, A.-R., Visconti, I., Wachsmann, C.: PUF-Enhanced RFID Security and Privacy. In: Secure Component and System Identification - SECSI 2010, Cologne, Germany (April 2010)

30. Singelée, D., Preneel, B.: Key Establishment Using Secure Distance Bounding Protocols. In: MOBIQUITOUS 2007: Proceedings of the 2007 Fourth Annual International Conference on Mobile and Ubiquitous Systems: Networking\&Services (MobiQuitous), Philadelphia, Pennsylvania, USA, pp. 1-6. IEEE Computer Society (August 2007)

31. Suh, G.E., Devadas, S.: Physical unclonable functions for device authentication and secret key generation. In: DAC 2007: Proceedings of the 44th Annual Design Automation Conference, pp. 9-14. ACM, New York (2007)

32. Trujillo-Rasua, R., Martin, B., Avoine, G.: The Poulidor Distance-Bounding Protocol. In: Ors Yalcin, S.B. (ed.) RFIDSec 2010. LNCS, vol. 6370, pp. 239-257. Springer, Heidelberg (2010)

33. Tu, Y.-J., Piramuthu, S.: RFID Distance Bounding Protocols. In: First International EURASIP Workshop on RFID Technology, Vienna, Austria (September 2007)

34. Tuyls, P., Batina, L.: RFID-Tags for Anti-counterfeiting. In: Pointcheval, D. (ed.) CT-RSA 2006. LNCS, vol. 3860, pp. 115-131. Springer, Heidelberg (2006)

35. Vaudenay, S.: On Privacy Models for RFID. In: Kurosawa, K. (ed.) ASIACRYPT 2007. LNCS, vol. 4833, pp. 68-87. Springer, Heidelberg (2007)

36. Čapkun, S., Buttyán, L., Hubaux, J.-P.: SECTOR: secure tracking of node encounters in multi-hop wireless networks. In: SASN 2003: Proceedings of the 1st ACM Workshop on Security of Ad Hoc and Sensor Networks, pp. 21-32. ACM, New York (2003)

37. Dodis, Y., Reyzin, L., Smith, A.: Fuzzy Extractors. In: Security with Noisy Data, pp. 79-99. Springer, Heidelberg (2007) 